\title{
Scalar meson and glueball decays within a effective chiral approach
}

\author{
F. Giacosa, Th. Gutsche, V.E. Lyubovitskij \\ and Amand Faessler \\ Institut für Theoretische Physik, Universität Tübingen, \\ Auf der Morgenstelle 14, D-72076 Tübingen, Germany
}

\begin{abstract}
We study the strong and electromagnetic decay properties of scalar mesons above $1 \mathrm{GeV}$ within a chiral approach. The scalar-isoscalar states are treated as mixed states of quarkonia and glueball configurations. A fit to the experimental decay rates listed by the Particle Data group is performed to extract phenomenological constraints on the nature of the scalar resonances. A comparison to other theoretical approaches in the scalar meson sector is discussed.
\end{abstract}

PACS: $12.39 . \mathrm{Fe}, 12.39 . \mathrm{Mk}, 13.25 . \mathrm{Jx}, 13.40 . \mathrm{Hg}$

Keywords: Scalar mesons, glueball, chiral Lagrangians, strong and radiative decays.

The interpretation of scalar mesons constitutes an unsolved problem of hadronic QCD. Below the mass scale of $2 \mathrm{GeV}$ various scalar states [1] are encountered: the isoscalar resonances $\sigma=f_{0}(400-1200), f_{0}(980), f_{0}(1370), f_{0}(1500)$ and $f_{0}(1710)$, the isovectors $a_{0}(980)$ and $a_{0}(1450)$ and the isodoublets $K_{0}^{*}(800)$ and $K_{0}^{*}(1430)$. The existence of the $K_{0}^{*}(800)$ is still not well established and omitted from the summary tables of [1]. From a theoretical point of view one expects the scalar quark-antiquark ground state nonet $0^{++}$, a scalar-isoscalar glueball, which lattice QCD predicts to be the lightest gluonic meson with a mass between 1.4-1.8 GeV [2], and possibly other exotic states (non $\bar{q}$ states), like e.g. four quark states or mesonic molecules [3]. Various interpretations of and assignments for the physical scalar resonances in terms of the expected theoretical states have been proposed (see, for instance, the review papers $[3,4]$ and References therein).

In this work we follow the original assignment of Ref. [5], where in a minimal scenario the bare quarkonium states $N \equiv \sqrt{1 / 2}(\bar{u} u+\bar{d} d)=\bar{n} n, S \equiv \bar{s} s$ and the bare scalar glueball $G$ mix, resulting in the three scalar-isoscalar resonances 
$f_{0}(1370), f_{0}(1500)$ and $f_{0}(1710)$. Such a mixing scheme has been previously investigated by many authors, as for example in the lattice study of [6] or within the model approaches of [7-11]. The mesons $a_{0}(1450)$ and $K_{0}^{*}(1430)$ are considered as the $I=1$ and $I=1 / 2$ quarkonia $J^{P C}=0^{++}$states. On the other hand, the scalars below $1 \mathrm{GeV}$ could be interpreted as four quark states or mesonic molecules.

In this Letter, starting from an effective chiral Lagrangian, we perform a treelevel analysis of the strong and electromagnetic decays of scalar mesons settled in the energy range between 1 and $2 \mathrm{GeV}$. Although a chiral approach cannot be rigorously justified at this energy scale, since loop corrections can be large, we intend to use this framework as a phenomenological tool to extract possible glueball-quarkonia mixing scenarios from the observed decays. This study is in line with the original application of Ref. [14], where detailed arguments can be found to justify this approach.

Chiral Lagrangian - The strong and electromagnetic decays of scalar mesons are based on the effective chiral Lagrangian $\mathcal{L}_{\text {eff }}$, derived in Chiral Perturbation Theory (ChPT) [12-14]. The Lagrangian involves the nonets of pseudoscalar $\left(\mathcal{P}=\sum_{i=0}^{8} P_{i} \lambda_{i} / \sqrt{2}\right)$ and of scalar mesons $\left(\mathcal{S}=\sum_{i=0}^{8} S_{i} \lambda_{i} / \sqrt{2}\right)$, the electromagnetic field and, in addition, a new degree of freedom, the bare glueball field $G$. We display the lowest order Lagrangian $\mathcal{L}_{\text {eff }}$, where the starting point is the large $N_{c}$ limit. Corrections to the large $N_{c}$ limit are encoded in $\mathcal{L}_{\text {mix }}^{P}$ and $\mathcal{L}_{\text {mix }}^{S}$.

$$
\begin{aligned}
\mathcal{L}_{\text {eff }} & =\frac{F^{2}}{4}\left\langle D_{\mu} U D^{\mu} U^{\dagger}+\chi_{+}\right\rangle+\frac{1}{2}\left\langle D_{\mu} \mathcal{S} D^{\mu} \mathcal{S}-M_{\mathcal{S}}^{2} \mathcal{S}^{2}\right\rangle+\frac{1}{2}\left(\partial_{\mu} G \partial^{\mu} G-M_{G}^{2} G^{2}\right) \\
& +c_{d}^{s}\left\langle\mathcal{S} u_{\mu} u^{\mu}\right\rangle+c_{m}^{s}\left\langle\mathcal{S} \chi_{+}\right\rangle+\frac{c_{d}^{g}}{\sqrt{3}} G\left\langle u_{\mu} u^{\mu}\right\rangle+\frac{c_{m}^{g}}{\sqrt{3}} G\left\langle\chi_{+}\right\rangle \\
& +c_{e}^{s}\left\langle\mathcal{S} F_{\mu \nu}^{+} F^{+\mu \nu}\right\rangle+\frac{c_{e}^{g}}{\sqrt{3}} G\left\langle F_{\mu \nu}^{+} F^{+\mu \nu}\right\rangle+\mathcal{L}_{\text {mix }}^{P}+\mathcal{L}_{\text {mix }}^{S}
\end{aligned}
$$

Here the symbol $\langle\ldots\rangle$ denotes the trace over flavor matrices. The constants $c_{d}^{s}$, $c_{m}^{s}, c_{d}^{g}, c_{m}^{g}, c_{e}^{s}$ and $c_{e}^{g}$ define the coupling of scalar fields and the bare glueball to pseudoscalar mesons and photons, respectively (the coupling constant $c_{e}^{g}$ is supposed to be suppressed because gluons do not couple directly to photons. However, an intermediate state of two vector mesons can in the framework of vector meson dominance generate a coupling of the glueball to the twophoton final state; this coupling is however not considered in the numerical analysis). The field $G$ is introduced as an extra flavor-singlet field in the effective lagrangian. We use the standard notation for the basic blocks of the ChPT Lagrangian [12]: $U=u^{2}=\exp (i \mathcal{P} \sqrt{2} / F)$ is the chiral field collecting pseudoscalar fields in the exponential parametrization, $D_{\mu}$ denotes the chiral and gauge-invariant derivative, $u_{\mu}=i u^{\dagger} D_{\mu} U u^{\dagger}, \quad \chi_{ \pm}=u^{\dagger} \chi u^{\dagger} \pm u \chi^{\dagger} u, \quad \chi=$ 
$2 B(s+i p), \quad s=\mathcal{M}+\ldots$ and $F_{\mu \nu}^{+}=\left(u^{\dagger} F_{\mu \nu} Q u+u F_{\mu \nu} Q u^{\dagger}\right)$, where $F_{\mu \nu}$ is the stress tensor of the electromagnetic field; $Q=\operatorname{diag}\{2 / 3,-1 / 3,-1 / 3\}$ and $\mathcal{M}=\operatorname{diag}\left\{\hat{m}, \hat{m}, m_{s}\right\}$ are the charge and the mass matrix of current quarks, respectively (we restrict to the isospin symmetry limit with $m_{u}=m_{d}=\hat{m}$ ); $B$ is the quark vacuum condensate parameter. Then the masses of the pseudoscalar mesons in the leading order of the chiral expansion are given by $M_{\pi}^{2}=2 \hat{m} B, M_{K}^{2}=\left(\hat{m}+m_{s}\right) B, M_{\eta^{8}}^{2}=(2 / 3)\left(\hat{m}+2 m_{s}\right) B$.

We intend to employ the Lagrangian (1) for the tree-level calculation of the two-pseudoscalar decays of scalar mesons. At the energy scale of interest, $E \sim M_{\mathcal{S}} \sim 1.5 \mathrm{GeV}$, a calculation of loops and an application of the power counting rules are not rigorously justified. The aim of the present approach is a phenomenological study of scalar meson physics, for which a tree-level calculation represents a useful analysis of the issue.

The term $\mathcal{L}_{\text {mix }}^{P} \sim \eta^{0} \eta^{8}$ gives rise to the singlet-octet mixing in the pseudoscalar and the scalar multiplets. The physical states $\eta$ and $\eta^{\prime}$ are given by

$$
\eta^{0}=\eta^{\prime} \cos \theta_{P}-\eta \sin \theta_{P}, \quad \eta^{8}=\eta^{\prime} \sin \theta_{P}+\eta \cos \theta_{P},
$$

where $\theta_{P}$ is the pseudoscalar mixing angle. We follow the standard procedure [13-15] and diagonalize the corresponding $\eta^{0}-\eta^{8}$ mass matrix to obtain the masses of $\eta$ and $\eta^{\prime}$. By using $M_{\pi}=139.57 \mathrm{MeV}, M_{K}=493.677 \mathrm{MeV}$ (the physical charged pion and kaon masses), $M_{\eta}=547.75 \mathrm{MeV}$ and $M_{\eta^{\prime}}=957.78$ $\mathrm{MeV}$ the mixing angle is determined as $\theta_{P}=-9.95^{\circ}$, which corresponds to the tree-level result (see details in Ref. [15]). Higher order corrections in ChPT cause a doubling of the absolute value of the pseudoscalar mixing angle [15]); in our work we restrict to the tree-level evaluation, we therefore consistently use the corresponding tree-level result of $\theta_{P}=-9.95^{\circ}$ (in the present approach we do not include the neutral pion when considering mixing in the pseudoscalar sector, because we work in the isospin limit; this mixing is small, and can be safely neglected when studying the decay of scalar resonances into two pseudoscalars). Similarly, for all pseudoscalar mesons we use the unified leptonic decay constant $F$, which is identified with the pion decay constant $F=F_{\pi}=92.4 \mathrm{MeV}$. A more accurate analysis including higher orders should use the individual couplings of the pseudoscalar mesons (for a detailed discussion see Refs. [16]).

Glueball-Quarkonia Mixing - We briefly discuss the mixing of the glueball with the scalar-isoscalar quarkonia states. The singlet $\left(S^{0}\right)$ and octet $\left(S^{8}\right)$ scalar quarkonia states are defined in terms of the nonstrange $N$ and strange $S$ components with the respective flavor content $[\bar{u} u+\bar{d} d] / \sqrt{2}$ and $\bar{s} s$ as

$$
S^{0}=\sqrt{2 / 3} N+\sqrt{1 / 3} S, \quad S^{8}=\sqrt{1 / 3} N-\sqrt{2 / 3} S
$$


In (1) the scalar nonet states have the same mass $M_{\mathcal{S}}$, corresponding to the flavor and large $N_{c}$ limits. Deviations from this configuration (i.e. from the large $N_{c}$ limit) are encoded in $\mathcal{L}_{\text {mix }}^{S}$ including quarkonia-glueball mixing leading to different masses for the scalar mesons (in [14] this is explicitly shown for the scalar nonet, but no glueball is included; the glueball mixing is also a deviation from large $N_{c}$, since in this limit the glueball and the quarkonia states decouple). As a result, the scalar-isoscalar sector can be described by the most general Klein-Gordon (KG) Lagrangian including mixing among the $N, S$ and the bare glueball $G$ configurations [5-9]:

$$
\mathcal{L}_{K G}=-\frac{1}{2} \sum_{\Phi=N, G, S} \Phi\left[\square+M_{\Phi}^{2}\right] \Phi-f G S-\sqrt{2} f r G N-\varepsilon N S,
$$

where $\square=\partial^{\mu} \partial_{\mu}$. The parameter $f$ is the quarkonia-glueball mixing strength, analogous to the parameter $z$ of Refs. [5-9]; $z$ refers to the quantum mechanical case where the mass matrix is linear in the bare mass terms, $f$ in turn is related to the the quadratic Klein-Gordon case. The connection between $f$ and $z$ discussed in Refs. $[8,11]$ leads to the approximate relation $f \simeq 2 z M_{G}$. If $r=1$, then the glueball is flavor blind and mixes only with the quarkonium flavor singlet $S^{0}$ (this is the case in Refs. [5,8,9]); a value $r \neq 1$ takes into account a possible deviation from this limit. However, as deduced from the analyses of Refs. $[6,7,11] r$ is believed to be close to unity. In the following we will use the limit $r=1$, i.e. we restrict to a flavor blind mixing.

The parameter $\varepsilon$ induces a direct mixing between $N$ and $S$ quarkonia states. This effect is neglected in [5,7-9], where flavor mixing is considered a higherorder effect. However, a substantial $N-S$ mixing in the scalar sector is the starting point of the analysis of Refs. $[17,18]$. The origin of quarkonia flavor mixing is, according to $[17,18]$, connected to instantons as in the pseudoscalar channel, but with opposite sign (see also [19]): the mixed physical fields are predicted to be a higher lying state of flavor structure $[N \sqrt{2}-S] / \sqrt{3}$ and a lower one with $[N+S \sqrt{2}] / \sqrt{3}$. Here we study the case $\varepsilon \neq 0$, more precisely $\varepsilon>0$, which leads to the same phase structure as in Ref. $[17,18]$, but the quantitative results and interpretation will differ. The physical scalar states $|i\rangle$ are identified with $i=f_{1} \equiv f_{0}(1370), f_{2} \equiv f_{0}(1500)$ and $f_{3} \equiv f_{0}(1710)$, which are set up as linear combinations of the bare states: $|i\rangle=B^{i N}|N\rangle+$ $B^{i G}|G\rangle+B^{i S}|S\rangle$. The amplitudes $B^{i j}$ are the elements of a matrix $B$ which diagonalizes the mass matrix of bare states including mixing, which in turn gives rise to the mass matrix of physical states $\Omega^{\prime}=\operatorname{diag}\left\{M_{f_{1}}^{2}, M_{f_{2}}^{2}, M_{f_{3}}^{2}\right\}$.

Results for suppressed glueball decays - In the following we determine a best fit of the parameters entering in Eqs. (1) and (3) to the experimental averages of masses and decay modes listed in Ref. [1]. Decay rates resulting from Eq. (1) are evaluated on the tree-level. First we analyze the case of a non-decaying glueball, i.e. $c_{d}^{g}=c_{m}^{g}=c_{e}^{g}=0$. In this scheme the decays are dominated 
by the quarkonia components [5] in line with large $N_{c}$ arguments. The phenomenological analysis of Ref. [9] confirmed this trend, but the recent fit of [7] shows a strong contribution by the direct decays of the glueball configuration. The strength of the glueball decays remains a point to clarify. Our results for suppressed glueball decays are given in Table 1 in comparison to the data of Ref. [1]. The only accepted average not included in the fit is $\Gamma_{f_{2} \rightarrow \eta \eta^{\prime}}$ for the reason that the decay channel $\eta \eta^{\prime}$ is produced at threshold, therefore a significant distortion due to the finite width of the state is expected. The state $f_{0}(1710)$ has only been observed in the decays into two pseudoscalar mesons [1]. The decay into the final state $4 \pi$, which can be fed by higher meson resonances, is suppressed [20]. We therefore impose the additional condition that the sum of partial decay widths into two pseudoscalar mesons $\left(\Gamma_{f_{3}}\right)_{2 P}$ saturates the total width $\left(\Gamma_{f_{3}}\right)_{t o t}$ with $\left(\Gamma_{f_{3}}\right)_{2 P}=\left(\Gamma_{f_{3}}\right)_{t o t}$ as indicated in Table 1 . Such a constraint is necessary to obtain meaningful total decay widths: without this condition on the full width a minimum for $\chi^{2}$ is obtained where $\left(\Gamma_{f_{3}}\right)_{t o t}$ is larger than $1 \mathrm{GeV}$, a clearly unacceptable solution. A local minimum for $\chi_{t o t}^{2}$ is found for the set of parameters

$$
\begin{aligned}
M_{N} & =1.455 \mathrm{GeV}, M_{G}=1.490 \mathrm{GeV}, M_{S}=1.697 \mathrm{GeV}, f=0.065 \mathrm{GeV}^{2}, \\
\varepsilon & =0.211 \mathrm{GeV}^{2}, c_{d}^{s}=8.48 \mathrm{MeV}, c_{m}^{s}=2.59 \mathrm{MeV} \chi_{\text {tot }}^{2}=29.01
\end{aligned}
$$

Table 1. Mass and decay properties of scalar $f_{0}$ mesons.

$\begin{array}{llll}\text { Quantity } & \text { Exp } & \text { Theory } & \chi_{i}^{2} \\ M_{f_{1}}(\mathrm{MeV}) & 1350 \pm 150 & 1417 & 0.202 \\ M_{f_{2}}(\mathrm{MeV}) & 1507 \pm 5 & 1507 & \sim 0 \\ M_{f_{3}}(\mathrm{MeV}) & 1714 \pm 5 & 1714 & 0.003 \\ \Gamma_{f_{2} \rightarrow \pi \pi}(\mathrm{MeV}) & 38.0 \pm 4.6 & 38.52 & 0.011 \\ \Gamma_{f_{2} \rightarrow \bar{K} K}(\mathrm{MeV}) & 9.4 \pm 1.7 & 10.36 & 0.322 \\ \Gamma_{f_{2} \rightarrow \eta \eta}(\mathrm{MeV}) & 5.6 \pm 1.4 & 1.90 & 8.109 \\ \Gamma_{f_{3} \rightarrow \pi \pi} / \Gamma_{f_{3} \rightarrow \bar{K} K} & 0.20 \pm 0.06 & 0.212 & 0.036 \\ \Gamma_{f_{3} \rightarrow \eta \eta} / \Gamma_{f_{3} \rightarrow \bar{K} K} & 0.48 \pm 0.15 & 0.249 & 2.446 \\ \Gamma_{a_{0} \rightarrow \bar{K} K} / \Gamma_{a_{0} \rightarrow \pi \eta} & 0.88 \pm 0.23 & 0.838 & 0.032 \\ \Gamma_{a_{0} \rightarrow \pi \eta^{\prime}} / \Gamma_{a_{0} \rightarrow \pi \eta} & 0.35 \pm 0.16 & 0.288 & 0.150 \\ \Gamma_{K^{*} \rightarrow K \pi}(\mathrm{MeV}) & 273 \pm 51 & 59.10 & 17.590 \\ \left(\Gamma_{f_{3}}\right)_{2 P}(\mathrm{MeV}) & 140 \pm 10 & 143.27 & 0.110 \\ \chi_{t o t}^{2} & - & - & 29.01\end{array}$


Implications of the fit - The bare non-strange quarkonia field $N$ has a mass of $M_{N}=1.455 \mathrm{GeV}$ which is, as desired, similar to the scale set by the isotriplet combination $a_{0}(1450)$ with a mass of $M_{a_{0}}=1.474 \pm 0.019 \mathrm{GeV}$ [1]. The mass of the bare glueball $M_{G}=1.490 \mathrm{GeV}$ is in agreement with the lattice results [2] and with the phenomenological analyses of $[5,7,9]$. The bare state $S$ has a mass of $M_{S}=1.697 \mathrm{GeV}$, which is about $\sim 200 \mathrm{MeV}$ heavier than the $N$ state, an acceptable mass difference like in the tensor meson nonet.

For the glueball-quarkonia mixing parameter we get $f=0.065 \mathrm{GeV}^{2}$, which by the approximate relation $f \simeq 2 z M_{G}[8,11]$ corresponds to $z \simeq 21.8 \mathrm{MeV}$. The results of Refs. $[7,9,11]$ are $z=85 \pm 10 \mathrm{MeV}, z=80 \mathrm{MeV}$ and $z \simeq 62 \mathrm{MeV}$, respectively, i.e. of the same order, but larger. The introduction of additional flavor mixing between the quarkonia configurations in the fit, as done here, leads to a reduction of the strength parameter $f$. The lattice result of Ref. [6] with $43 \pm 31 \mathrm{MeV}$ is in agreement with the present evaluation, but has a large uncertainty.

The flavor mixing parameter resulting from the fit is $\varepsilon=0.211 \mathrm{GeV}^{2}$. In the limit $f=0$ the mixed physical states are $\left|f_{1}\right\rangle=0.97|N\rangle+0.26|S\rangle$ and $\left|f_{3}\right\rangle=-0.26|N\rangle+0.97|S\rangle$ (and, of course, $\left|f_{2}\right\rangle=|G\rangle$ ). The phase structure of the mixed states is, as discussed previously, as in [17-19]. But here the strength of flavor mixing is smaller, resulting in mixed states which are dominantly $N$ or $S$. The influence however of (an even small) flavor mixing in strong and electromagnetic decays may be non-negligible.

The mixing matrix $B$ relating the physical to the bare states in the present fit is expressed as:

$$
\left(\begin{array}{l}
\left|f_{1}\right\rangle \equiv\left|f_{0}(1370)\right\rangle \\
\left|f_{2}\right\rangle \equiv\left|f_{0}(1500)\right\rangle \\
\left|f_{3}\right\rangle \equiv\left|f_{0}(1710)\right\rangle
\end{array}\right)=\left(\begin{array}{ccc}
0.86 & 0.45 & 0.24 \\
-0.45 & 0.89 & -0.06 \\
-0.24 & -0.06 & 0.97
\end{array}\right)\left(\begin{array}{l}
|N\rangle \equiv|\bar{n} n\rangle \\
|G\rangle \equiv|g g\rangle \\
|S\rangle \equiv|\bar{s} s\rangle
\end{array}\right) .
$$

The physical resonances are dominated by the diagonal bare components, qualitatively in line with Refs. $[3,5,7,9]$. Since the glueball does not contribute to the decay, the relative phase with respect to the quarkonia components is at this stage irrelevant; by inverting $f \rightarrow-f$ we would find the same results for the decays, but opposite glueball-quarkonia phases. In turn, the relative phases of the $N$ and $S$ components are not symmetric under $\varepsilon \rightarrow-\varepsilon$; as discussed above, in $f_{0}(1370)$ they are in phase, while in $f_{0}(1710)$ they are out of phase. The state $\left|f_{0}(1500)\right\rangle$ behaves like a $N$ state with a decreased width, while the $\mathrm{S}$ component is small $[9,21]$. Thus the decay into $K \bar{K}$ is smaller than the $\pi \pi$ channel. The $N$ and $S$ state components are in phase contrary to the results of $[5,9]$. 
The experimental uncertainties of the $f_{0}(1370)$ resonance are large, no average or fit is presented in [1]. The main problem connected with this resonance is its large width (200-500 $\mathrm{MeV}$ ) and its partial overlap with the broad low-lying $\sigma \equiv f_{0}(400-1200)$. However, the results from WA102 [20] indicate a large $N$ component in its wave function; the results from Crystal Barrel (summarized in [22] and subsequently analyzed in [23]) confirm such a trend (see also [24] for a recent review).

Table 2. Decays of $f_{1}=f_{0}(1370)$.

$\begin{array}{lll}\text { Quantity } & \operatorname{Exp}(\text { WA102) } & \text { Theory } \\ \Gamma_{f_{1} \rightarrow \bar{K} K} / \Gamma_{f_{1} \rightarrow \pi \pi} & 0.46 \pm 0.19 & 0.34 \\ \Gamma_{f_{1} \rightarrow \eta \eta} / \Gamma_{f_{1} \rightarrow \pi \pi} & 0.16 \pm 0.07 & 0.06 \\ \left(\Gamma_{f_{1}}\right)_{2 P}(\mathrm{MeV}) & \text { "small" } & 166\end{array}$

Predictions for the two-pseudoscalar decay modes are in acceptable agreement with the results of WA102 as shown in Table 2. The measured ratio $\Gamma_{f_{1} \rightarrow 4 \pi} / \Gamma_{f_{1} \rightarrow \pi \pi}=34.0_{-9}^{+22}[20]$, although the errors are large, points to a dominant $4 \pi$ contribution to the total width. Our prediction gives however a sizable contribution of the two-pseudoscalar decay mode.

An analysis by Crystal Barrel [23] also indicates sizable partial decay widths of the $4 \pi$ decay channels: $\Gamma_{f_{1} \rightarrow \sigma \sigma}=120.5 \pm 65 \mathrm{MeV}$ and $\Gamma_{f_{1} \rightarrow \rho \rho}=62.2 \pm$ $28.8 \mathrm{MeV}$. The same analysis gives the following two-pseudoscalar partial widths $[23,24]: \Gamma_{f_{1} \rightarrow \pi \pi}=21.7 \pm 9.9 \mathrm{MeV}, \Gamma_{f_{1} \rightarrow K \bar{K}}=(7.9 \pm 2.7 \mathrm{MeV})$ to $(21.2 \pm 7.2 \mathrm{MeV}), \Gamma_{f_{1} \rightarrow \eta \eta}=0.41 \pm 0.27 \mathrm{MeV}$. On the contrary, the analysis of [25] reports the ratio $\Gamma_{f_{1} \rightarrow \pi \pi} /\left(\Gamma_{f_{1}}\right)_{\text {tot }}=0.26 \pm 0.09$, which is in disagreement with the previously discussed experimental results.

In the original work [5], a quarkonium state $N$ has a very large two-pseudoscalar width with $\left(\Gamma_{N}\right)_{2 P} \sim 500 \mathrm{MeV}$. Since the $f_{0}(1370)$ is interpreted as a dominant $N$ state [5] one also expects a large value for $\left(\Gamma_{f_{1}}\right)_{2 P}$. A sizable value for $\left(\Gamma_{N}\right)_{2 P}$ is also predicted in [9]; for the mixed state $f_{0}(1370)$ one has $\left(\Gamma_{f_{1}}\right)_{2 P}=115.7 \mathrm{MeV}$ comparable to the present result. In a recent fit [7] small two-pseudoscalar partial decay widths are obtained by the following mechanism: for $f_{0}(1370)$ the glueball component gives large decay amplitudes which interfere destructively with the contribution of the $N$ component. As a result [7], the two-pseudoscalar decay width $\left(\Gamma_{f_{1}}\right)_{2 P}$ is smaller than $\left(\Gamma_{f_{2}}\right)_{2 P}$ with $\left(\Gamma_{f_{2}}\right)_{2 P} /\left(\Gamma_{f_{1}}\right)_{2 P}=10.0 \pm 3.0$; at the same time $\left(\Gamma_{f_{3}}\right)_{2 P} /\left(\Gamma_{f_{2}}\right)_{2 P}=0.7 \pm 0.2$. In the present fit with a suppressed glueball decay we have the following decay widths into two pseudoscalar pairs: $\left(\Gamma_{f_{1}}\right)_{2 P}=165.79 \mathrm{MeV}>\left(\Gamma_{f_{3}}\right)_{2 P}=143.27$ $\mathrm{MeV}>\left(\Gamma_{f_{2}}\right)_{2 P}=50.82 \mathrm{MeV}$. The experimental situation concerning the absolute scale of the partial decay widths into pseudoscalars remains unclear, 
but the explicit values constitute a key test of mixing scenarios.

The theoretical partial widths of the $f_{0}(1500)$ are in good agreement with the data (see Table 1) apart from a slight underestimate of the $2 \eta$ channel. We also obtain $\Gamma_{f_{2} \rightarrow \eta \eta^{\prime}}=0.036 \mathrm{MeV}$ as compared to the experimental value of $\Gamma_{f_{2} \rightarrow \eta \eta^{\prime}}=2 \pm 1 \mathrm{MeV}$. Taking into account the finite width of the resonance will lead to an increase of the theoretical value.

For the decays of $f_{0}(1710)$ we summarize our results compared to the data of WA102 [20] in Table 3.

Table 3. Decays of $f_{3}=f_{0}(1710)$.

$$
\begin{array}{lll}
\text { Quantity } & \operatorname{Exp}(\text { WA102) } & \text { Theory } \\
\Gamma_{f_{3} \rightarrow \bar{K} K} / \Gamma_{f_{3} \rightarrow \pi \pi} & 5.0 \pm 0.7 & 4.70 \\
\Gamma_{f_{3} \rightarrow \eta \eta} / \Gamma_{f_{3} \rightarrow \pi \pi} & 2.4 \pm 0.6 & 1.17 \\
\Gamma_{f_{3} \rightarrow \eta \eta^{\prime}} / \Gamma_{f_{3} \rightarrow \pi \pi} & <0.18 & 1.59 \\
\left(\Gamma_{f_{3}}\right)_{2 P}(\mathrm{MeV}) & \text { "dominant" } & 143.27
\end{array}
$$

The first two ratios, already included in the fit of Table 2, can be reproduced. The theoretical ratio $\Gamma_{f_{3} \rightarrow \eta \eta^{\prime}} / \Gamma_{f_{3} \rightarrow \pi \pi}$, which is not included in [1], is in complete disagreement with the WA102 result. The dominance of the $\eta \eta^{\prime}$ mode over $\pi \pi$ is a solid prediction, which does not depend very much on the choice of parameters. A confirmation of the experimental result could possibly hint at a sizable role of direct glueball decay.

The ratios of two-pseudoscalar decay modes of $a_{0}(1450)$, included in the fit of Table 2, are well reproduced. The prediction for the two-pseudoscalar width of $\left(\Gamma_{a_{0}}\right)_{2 P}=84.26 \mathrm{MeV}$ is smaller than the total width of $265 \pm 13 \mathrm{MeV}$. However, the experimental ratio $\left(\Gamma_{a_{0} \rightarrow \omega \pi \pi} / \Gamma_{a_{0} \rightarrow \pi \eta}\right)$ is not known: no average or fit is listed in [1]. The experimental value from [26], which is $10.7 \pm 2.3$, would imply a dominant $\omega \pi \pi$ mode and in turn rather small two-pseudoscalar partial decay widths. This finding is in disagreement with the results of $[5,27]$. In [5] a value of $\left(\Gamma_{a_{0}}\right)_{2 P}=390 \pm 110 \mathrm{MeV}$ is found, in the work of [27] one has $\left(\Gamma_{a_{0}}\right)_{2 P}=420-940 \mathrm{MeV}$ for $a_{0}$ masses in the range of 1200-1400 $\mathrm{MeV}$. For our value for $\left(\Gamma_{a_{0}}\right)_{2 P}$ we obtain the estimate: $\Gamma_{a_{0} \rightarrow \omega \pi \pi} / \Gamma_{a_{0} \rightarrow \pi \eta}=$ $\left[\left(\Gamma_{a_{0}}\right)_{t o t}-\left(\Gamma_{a_{0}}\right)_{2 P}\right] / \Gamma_{a_{0} \rightarrow \pi \eta} \sim 4.5$.

Our result for $\Gamma_{K^{*} \rightarrow K \pi}$ underestimates the experimental value by about a factor of 5 (see Table 1). Furthermore, for the additional $K \eta$ decay channel we get $\Gamma_{K^{*} \rightarrow K \eta} / \Gamma_{K^{*} \rightarrow \pi K}=0.026$.

In [27] a value of $\Gamma_{K^{*} \rightarrow K \pi}=340 \mathrm{MeV}$ is predicted, but, as discussed above, 
$\left(\Gamma_{a_{0}}\right)_{2 P}$ is of the order of $1 \mathrm{GeV}$, much larger than the full width. Similarly, in [5] with $\Gamma_{K^{*} \rightarrow K \pi}=200 \pm 20$ and $\left(\Gamma_{a_{0}}\right)_{2 P}=390 \pm 110 \mathrm{MeV}$ the first result underestimates while the second overshoots the experimental value. A full analysis in the ${ }^{3} P_{0}$ model [28] results in $\Gamma_{K^{*} \rightarrow \pi K}=166 \mathrm{MeV}, \Gamma_{N \rightarrow \pi \pi}=271 \mathrm{MeV}$. Unfortunately, the resonance $a_{0}(1450)$ is not discussed in [28]. The authors of [28] also tried to fix $\Gamma_{K^{*} \rightarrow \pi K}$ to its experimental value, and then calculate the $2 \pi$ partial width of a $N$ state, obtaining $\Gamma_{N \rightarrow \pi \pi} \sim 450 \mathrm{MeV}$. The last result implies a very large two-pseudoscalar and full width for a $N$ state. A full experimental determination of all relevant decay modes involving $a_{0}(1450)$ and $K_{0}^{*}(1430)$ would certainly help to clarify this issue.

If $f_{0}(1370)$ is dominantly $\bar{n} n$, as in $[5-9,11]$, there is, as discussed above, an incompatibility of the present experimental small two-pseudoscalar partial decay widths [23] and various model calculations. At the same time, the consistent understanding of the isodoublet states $K^{*}(1450)$, the isovectors $a_{0}(1450)$ is still incomplete.

Proceeding as in a [28], that is fitting $\Gamma_{K^{*} \rightarrow \pi K}$ (together with the ratios of $a_{0}(1450)$ ) we find $c_{d}^{s}=0.0180 \mathrm{GeV}$ (larger than a factor 2 when compared to the previous fit in Table 1) and $c_{m}^{s}=0.0073 \mathrm{GeV}$. With these values one has $\Gamma_{K^{*} \rightarrow \pi K}=281 \mathrm{MeV}, \Gamma_{a_{0} \rightarrow K \bar{K}} / \Gamma_{a_{0} \rightarrow \pi \eta}=0.88$ and $\Gamma_{a_{0} \rightarrow \pi \eta^{\prime}} / \Gamma_{a_{0} \rightarrow \pi \eta}=0.30$ and $\Gamma_{a_{0} \rightarrow 2 P}=381.17 \mathrm{MeV}$. The corresponding decay width of the $N$ state into two pseudoscalars is $\Gamma_{N \rightarrow 2 P}=937 \mathrm{MeV}$ (for a mass of $M_{N}=1.448 \mathrm{GeV}$ ). We then find similar results as in $[5,28]$, implying that the discussed trend is rather model independent. Fitting the remaining free parameters to the experimental data, a $\chi^{2}$ minimum results in: $M_{N}=1.453 \mathrm{GeV}, M_{G}=1.504$ $\mathrm{GeV}, M_{S}=1.698 \mathrm{GeV}, f=0.0281 \mathrm{GeV}^{2}, \varepsilon=0.209 \mathrm{GeV}^{2}$. The mixing parameter $f$ is much smaller than in the full fit, in disagreement with the results of $[5,7,9]$. As a further consequence the state $\left|f_{1}\right\rangle \simeq|N\rangle$ has a very large two-pseudoscalar width of about $1 \mathrm{GeV}$. At the same time $\left|f_{3}\right\rangle \simeq|S\rangle$, which also has a large (and not acceptable) two-pseudoscalar width of $\sim 700 \mathrm{MeV}$.

As a further consequence we discuss the two-photon decay rates of the scalar resonances. We assume that the coupling $c_{e}^{g}$ is suppressed with respect to $c_{e}^{s}$, i.e. we set the glueball-photon coupling $c_{e}^{g}$ to zero. The ratios of radiative decay widths as a prediction of the fit are:

$$
\Gamma_{f_{1} \rightarrow 2 \gamma}: \Gamma_{f_{2} \rightarrow 2 \gamma}: \Gamma_{f_{3} \rightarrow 2 \gamma}: \Gamma_{a_{0}^{0} \rightarrow 2 \gamma}=1: 0.367: 0.004: 0.491,
$$

which are independent of the coupling $c_{e}^{s}$. The result for $\Gamma_{f_{2} \rightarrow 2 \gamma} / \Gamma_{f_{1} \rightarrow 2 \gamma}$ is in qualitative agreement with the results of $[7,11]$. The ratio $\Gamma_{f_{3} \rightarrow 2 \gamma} / \Gamma_{f_{1} \rightarrow 2 \gamma}$, however, is considerably smaller than in the previous works. The suppression of $\Gamma_{f_{3} \rightarrow 2 \gamma}$ originates from the destructive interference between the $N$ and $S$ components, which in turn is traced to the flavor mixing with $\varepsilon>0$ in accord with the phases of $[17,18]$. Another interesting prediction is the ratio 
$\Gamma_{a_{0}^{0} \rightarrow 2 \gamma} / \Gamma_{f_{1} \rightarrow 2 \gamma}$, which is relatively large.

The experimental status of the two-photon decays is still incomplete. For the $f_{0}(1370)$ two values are indicated in PDG2000 [29]: $3.8 \pm 1.5 \mathrm{keV}$ and $5.4 \pm 2.3$ $\mathrm{keV}$. However, it is not clear if the two-photon signal comes from the $f_{0}(1370)$ or from the high mass end of the broad $f_{0}(400-1200)$. The PDG currently [1] seems to favor this last possibility, but the data could also be valid for the $f_{0}(1370)$. We therefore interpret the two experimental values as an upper limit for the two-photon decay width of the $f_{0}(1370)$. Signals for two-photon decays of $f_{0}(1500)$ and $f_{0}(1710)$ have not yet been seen; the following upper limits are reported [1]:

$$
\begin{gathered}
\Gamma_{f_{0}(1500) \rightarrow 2 \gamma}\left(\Gamma_{f_{0}(1500) \rightarrow \pi \pi} / \Gamma_{f_{0}(1500) t o t}\right)<0.46 \mathrm{keV}, \\
\Gamma_{f_{0}(1710) \rightarrow 2 \gamma}\left(\Gamma_{f_{0}(1710) \rightarrow K \bar{K}} / \Gamma_{f_{0}(1710) t o t}\right)<0.11 \mathrm{keV} .
\end{gathered}
$$

Using the known branching ratio $\Gamma_{f_{0}(1500) \rightarrow 2 \pi} / \Gamma_{f_{0}(1500) t o t}$ one gets $\Gamma_{f_{0}(1500) \rightarrow 2 \gamma}<$ $1.4 \mathrm{keV}$ [21]. An accepted fit for $\Gamma_{f_{0}(1710) \rightarrow K \bar{K}} / \Gamma_{f_{0}(1710) t o t}$ is not reported in [1]. Using the value from [30] with $\Gamma_{f_{0}(1710) \rightarrow K \bar{K}} / \Gamma_{f_{0}(1710) t o t}=0.38_{-0.13}^{+0.03}$ we find an upper limit of the order of $\Gamma_{f_{0}(1710) \rightarrow 2 \gamma} \sim 0.3 \mathrm{keV}$.

For an absolute prediction of the two-photon decay widths we use $c_{e}^{s}=0.0761$ $\mathrm{GeV}^{-1}$ as determined in the model approach of Ref. [11]. For the non-strange quarkonium state we get $\Gamma_{N \rightarrow 2 \gamma}=0.904 \mathrm{keV}$, while for the isovector and mixed scalars we have:

$$
\begin{aligned}
\Gamma_{f_{1} \rightarrow 2 \gamma} & =0.702 \mathrm{keV}, \Gamma_{f_{2} \rightarrow 2 \gamma}=0.258 \mathrm{keV}, \\
\Gamma_{f_{3} \rightarrow 2 \gamma} & =0.004 \mathrm{keV}, \Gamma_{a_{0}^{0} \rightarrow 2 \gamma}=0.345 \mathrm{keV} .
\end{aligned}
$$

The results for the mixed states are below the current upper limits.

The estimate for the $2 \gamma$ decay of the bare quarkonium state $\bar{n} n$ of $0.904 \mathrm{keV}$ is smaller than the one of [21], where the following expression has been used:

$$
\Gamma_{N \rightarrow 2 \gamma}\left(0^{++}\right)=k\left(\frac{M_{N}\left(0^{++}\right)}{M_{N}\left(2^{++}\right)}\right)^{3} \Gamma_{N \rightarrow 2 \gamma}\left(2^{++}\right)
$$

The coefficient $k$ is $15 / 4$ in a non-relativistic calculation, but becomes smaller when considering relativistic corrections [31]. In [21] a range of values for $k$ from 2 to $15 / 4$ is considered. Our chiral Lagrangian approach combined with [11] points to a smaller value of $k$; using our result for $\Gamma_{N \rightarrow 2 \gamma}\left(0^{++}\right)$and taking the value $\Gamma_{N \rightarrow 2 \gamma}\left(2^{++}\right)=2.60 \pm 0.24 \mathrm{keV}$ [1] at $M_{N}\left(2^{++}\right)=1.27 \mathrm{GeV}$ we get $k \sim 0.25$. This result is model dependent, since it relies on the parameters for the covariant description of the scalar mesons used in [11]. Hence a fully covariant treatment implies strong deviations from the non-relativistic limit. 
Results when including glueball decays - In line with strong coupling arguments [5] direct glueball decay can be suppressed, leading to the phenomenology discussed previously. Including explicit glueball decay in a full fit to the data points of Table 1 with nine parameters (three bare masses, two mixing parameters $f$ and $\epsilon$, four decay strengths $\left.c_{d}^{s}, c_{m}^{s}, c_{d}^{g}, c_{m}^{g}\right)$ does not lead to a meaningful and unique $\chi^{2}$ minimum. To study the effect of direct glueball decay in the context of an effective Lagrangian, we pursue following strategy: we keep the bare masses listed in (4), which are in accord with other phenomenological considerations, and only fit the mixing parameters and decay strengths.

We first consider the $S U(3)$ flavor symmetry limit for the direct glueball decay, that is $c_{m}^{g}=0$. A fit to the data of Table 1 results in the parameter values: $f=0.0650 \mathrm{GeV}^{2}, \varepsilon=0.211 \mathrm{GeV}^{2}, c_{d}^{s}=8.50 \mathrm{MeV}, c_{m}^{s}=2.58 \mathrm{MeV}$ and $c_{d}^{g}=$ $-0.004 \mathrm{MeV}$ with $\chi^{2}=29.00$. The flavor symmetric glueball decay constant $c_{d}^{g}$ is very small in this fit, whereas the other parameters are practically unchanged resulting in a $\chi^{2}$ only sligthly smaller than before.

Flavor symmetry breaking in the direct glueball decay is encoded in the strength parameter $c_{m}^{g}$. Inclusion of this additional parameter in a full fit results in the set of parameters: $f=0.0654 \mathrm{GeV}^{2}, \varepsilon=0.203 \mathrm{GeV}^{2}, c_{d}^{s}=8.90$ $\mathrm{MeV}, c_{m}^{s}=0.87 \mathrm{MeV}, c_{d}^{g}=0.42 \mathrm{MeV}$ and $c_{m}^{g}=-1.17 \mathrm{MeV}$ with $\chi^{2}=28.83$. The dominant parameters $f, \varepsilon$ and $c_{d}^{s}$ remain stable, when including the direct glueball decay. The coupling $c_{m}^{s}$ decreases slightly, whereas the glueball coupling $c_{d}^{g}$ remains suppressed and is smaller than $c_{m}^{g}$. Latter effect might suggest a strong flavor symmetry breaking when discussing direct glueball decays. Again, the resulting masses of the scalar-isoscalar mesons and decay rates listed in Table 1 remain essentially unchanged for a pure fit to the data. Without further guidance from theory concerning the direct glueball decay the present phenomenological fit seems to indicate that this decay modes are suppressed in line with the original arguments given in [5]. This result, however, contradicts the approach chosen in [7], where direct glueball decays dominate.

In the context of an effective chiral Lagrangian, evaluated at tree level, we discussed the phenomenological consequences for the scalar meson sector. Thereby, the scalar glueball is assumed to mix minimally with the scalarisoscalar quarkonia states. A fit of the mixing parameters and coupling strengths entering in this scheme to the accepted data yields several results: the direct flavor mixing between quarkonia states [17] leads to a structure of the physical $f_{0}$ states, which differs from the conventional scheme as in Refs. [7, 9]. The different pictures can be directly tested in radiative $2 \gamma$ decays of the $f_{0}$ mesons. The relevance of direct glueball decays, which play a dominant role in [7], cannot be verified in the present scheme. Further input from lattice simulations, as for example demonstrated in Ref. [32], could serve to obtain a better understanding of this issue. 


\section{Acknowledgments}

This work was supported by the DFG under contracts FA67/25-3, GRK683. This research is also part of the EU Integrated Infrastructure Initiative Hadronphysics project under contract number RII3-CT-2004-506078 and President grant of Russia "Scientific Schools" No.1743.2003.

\section{References}

[1] S. Eidelman et al. [Particle Data Group Collaboration], Phys. Lett. B 592, 1 (2004).

[2] C. Michael, arXiv:hep-lat/0302001; G. S. Bali, K. Schilling, A. Hulsebos, A. C. Irving, C. Michael and P. W. Stephenson [UKQCD Collaboration], Phys. Lett. B 309, 378 (1993) [arXiv:hep-lat/9304012]; G. S. Bali et al. [TXL Collaboration], Phys. Rev. D 62, 054503 (2000) [arXiv:hep-lat/0003012]; C. Morningstar and M. J. Peardon, AIP Conf. Proc. 688, 220 (2004) [arXiv:nucl-th/0309068].

[3] C. Amsler and N. A. Tornqvist, Phys. Rept. 389, 61 (2004).

[4] F. E. Close and N. A. Tornqvist, J. Phys. G 28, R249 (2002) [arXiv:hepph/0204205]; E. Klempt, arXiv:hep-ex/0101031; S. F. Tuan, arXiv:hepph/0303248; G. V. Efimov and M. A. Ivanov, "The Quark Confinement Model of Hadrons", IOP Publishing, Bristol \& Philadelphia, 1993.

[5] C. Amsler and F. E. Close, Phys. Lett. B 353, 385 (1995) [arXiv:hepph/9505219]; C. Amsler and F. E. Close, Phys. Rev. D 53, 295 (1996) [arXiv:hep-ph/9507326].

[6] W. J. Lee and D. Weingarten, Phys. Rev. D 61, 014015 (2000) [arXiv:heplat/9910008]; D. Weingarten, Nucl. Phys. Proc. Suppl. 53, 232 (1997) [arXiv:hep-lat/9608070].

[7] F. E. Close and A. Kirk, Eur. Phys. J. C 21, 531 (2001) [arXiv:hep-ph/0103173].

[8] L. Burakovsky and P. R. Page, Phys. Rev. D 59, 014022 (1999) [Erratum-ibid. D 59, 079902 (1999)] [arXiv:hep-ph/9807400].

[9] M. Strohmeier-Presicek, T. Gutsche, R. Vinh Mau and A. Faessler, Phys. Rev. D 60, 054010 (1999) [arXiv:hep-ph/9904461].

[10] M. A. Ivanov and R. K. Muradov, JETP Lett. 42, 367 (1985); M. Jaminon and B. van den Bossche, Nucl. Phys. A 636, 113 (1998) [arXiv:nucl-th/9712029]; J. V. Burdanov and G. V. Efimov, Phys. Rev. D 64, 014001 (2001) [arXiv:hepph/0009027]; M. K. Volkov and V. L. Yudichev, Eur. Phys. J. A 10, 223 (2001) [arXiv:hep-ph/0103003]. 
[11] F. Giacosa, T. Gutsche and A. Faessler, Phys. Rev. C 71, 025202 (2005) [arXiv:hep-ph/0408085].

[12] S. Weinberg, Physica A 96 (1979) 327; J. Gasser and H. Leutwyler, Annals Phys. 158, 142 (1984); Nucl. Phys. B 250 (1985) 465.

[13] G. Ecker, J. Gasser, A. Pich and E. de Rafael, Nucl. Phys. B 321, 311 (1989); G. Ecker, J. Gasser, H. Leutwyler, A. Pich and E. de Rafael, Phys. Lett. B 223, 425 (1989).

[14] V. Cirigliano, G. Ecker, H. Neufeld and A. Pich, JHEP 0306 (2003) 012 [arXiv:hep-ph/0305311].

[15] E. P. Venugopal and B. R. Holstein, Phys. Rev. D 57, 4397 (1998) [arXiv:hep$\mathrm{ph} / 9710382]$.

[16] J. Gasser and H. Leutwyler, Nucl. Phys. B 250 (1985) 465; R. Kaiser and H. Leutwyler, Eur. Phys. J. C 17 (2000) 623 [arXiv:hep-ph/0007101]; T. Feldmann, Int. J. Mod. Phys. A 15, 159 (2000) [arXiv:hep-ph/9907491].

[17] P. Minkowski and W. Ochs, Nucl. Phys. Proc. Suppl. 121, 123 (2003) [arXiv:hep-ph/0209225]; P. Minkowski and W. Ochs, arXiv:hep-ph/9905250.

[18] P. Minkowski and W. Ochs, Eur. Phys. J. C 9, 283 (1999) [arXiv:hep$\mathrm{ph} / 9811518]$.

[19] E. Klempt, B. C. Metsch, C. R. Munz and H. R. Petry, Phys. Lett. B 361, 160 (1995) [arXiv:hep-ph/9507449].

[20] D. Barberis et al. [WA102 Collaboration], Phys. Lett. B 479, 59 (2000) [arXiv:hep-ex/0003033].

[21] C. Amsler, Phys. Lett. B 541, 22 (2002) [arXiv:hep-ph/0206104].

[22] C. Amsler, Rev. Mod. Phys. 70, 1293 (1998) [arXiv:hep-ex/9708025].

[23] A. Abele et al. [CRYSTAL BARREL Collaboration], Eur. Phys. J. C 21, 261 (2001).

[24] U. Thoma, Eur. Phys. J. A 18, 135 (2003).

[25] D. V. Bugg, B. S. Zou and A. V. Sarantsev, Nucl. Phys. B 471, 59 (1996).

[26] C. A. Baker et al., Phys. Lett. B 563, 140 (2003).

[27] C. Gobbi, F. Iachello and D. Kusnezov, Phys. Rev. D 50, 2048 (1994) [arXiv:hep-ph/9310250].

[28] E. S. Ackleh, T. Barnes and E. S. Swanson, Phys. Rev. D 54, 6811 (1996) [arXiv:hep-ph/9604355].

[29] D. E. Groom et al. [Particle Data Group Collaboration], Eur. Phys. J. C 15, 1 (2000).

[30] R. S. Longacre et al., Phys. Lett. B 177, 223 (1986). 
[31] Z. P. Li, F. E. Close and T. Barnes, Phys. Rev. D 43, 2161 (1991).

[32] J. Sexton, A. Vaccarino and D. Weingarten, Nucl. Phys. Proc. Suppl. 47, 128 (1996) [arXiv:hep-lat/9602022]. 